\title{
Vacuum polarization in the Schwarzschild spacetime and dimensional reduction
}

\author{
R. Balbinot, ${ }^{1, *}$ A. Fabbri, ${ }^{1, \dagger}$ P. Nicolini, ${ }^{1, \$}$ V. Frolov, ${ }^{2, \S}$ P. Sutton, ${ }^{3, \|}$ and A. Zelnikov, ${ }^{2,4, \pi}$ \\ ${ }^{1}$ Dipartimento di Fisica dell'Università di Bologna and INFN sezione di Bologna, Via Irnerio 46, 40126 Bologna, Italy \\ ${ }^{2}$ Theoretical Physics Institute, Department of Physics, University of Alberta, Edmonton, Alberta, Canada T6G $2 \mathrm{~J} 1$ \\ ${ }^{3}$ Department of Physics, The Pennsylvania State University, State College, Pennsylvania 16802-6300 \\ ${ }^{4}$ P.N. Lebedev Physics Institute, Leninsky pr. 53, Moscow 117924, Russia
}

(Received 7 December 2000; published 28 March 2001)

\begin{abstract}
A massless scalar field minimally coupled to gravity and propagating in Schwarzschild spacetime is considered. After dimensional reduction under spherical symmetry the resulting 2D field theory is canonically quantized and the renormalized expectation values $\left\langle T_{a b}\right\rangle$ of the relevant energy-momentum tensor operator are investigated. Asymptotic behaviors and analytical approximations are given for $\left\langle T_{a b}\right\rangle$ in the Boulware, Unruh and Hartle-Hawking states. Special attention is devoted to the black-hole horizon region where the WKB approximation breaks down.
\end{abstract}

DOI: 10.1103/PhysRevD.63.084029

PACS number(s): 04.62.+v, 11.10.Gh, 11.10.Kk

\section{INTRODUCTION}

In quantum field theory the dimensional reduction of a system obeying some symmetries, such as spherical symmetry, is obtained by decomposing the field operators in harmonics in the symmetrical subspace. In the case of spherical symmetry, decomposing in terms of spherical harmonics effectively reduces a $4 \mathrm{D}$ theory to a set of $2 \mathrm{D}$ theories characterized by different values of the angular momentum.

Two-dimensional theories are often regarded as useful tools for inferring general features of systems whose behavior is sophisticated and difficult to analyze in the physical 4D spacetime. In some spherically symmetric systems the main physical effects come from the " $s$-wave sector" - the $l=0$ mode. Truncation of higher momentum modes is then obtained by integrating over the "irrelevant" angular variables. This is the spirit which pervades most of the vast literature on 2D black holes, though this s-wave approximation is not always accurate enough. These models are believed to describe the $s$-wave sector of physical 4D black holes.

Within this perspective, a model of $2 \mathrm{D}$ conformally invariant matter fields interacting with $2 \mathrm{D}$ dilaton gravity has attracted considerable interest recently. The action for this theory is

$$
S=-\frac{1}{2} \int d^{2} x \sqrt{-g} e^{-2 \phi} g^{a b} \partial_{a} \varphi \partial_{b} \varphi
$$

where $\varphi$ is the scalar field, $\phi$ the dilaton, $g_{a b}$ the $2 \mathrm{D}$ background metric and $a, b=1,2$.

The reason for this interest lies in the following: the action (1.1) can be obtained by the dimensional reduction of

\footnotetext{
*Electronic address: balbinot@bo.infn.it

†Electronic address: fabbria@bo.infn.it

*Electronic address: nicolini@bo.infn.it

${ }^{\S}$ Electronic address: frolov@phys.ualberta.ca

"Electronic address: psutton@ gravity.phys.psu.edu

${ }^{\mathbb{I}}$ Electronic address: zelnikov@phys.ualberta.ca
}

the 4D action for a massless scalar field minimally coupled to $4 \mathrm{D}$ gravity,

$$
S^{(4)}=-\frac{1}{8 \pi} \int d^{4} x \sqrt{-g^{(4)}} g^{\mu \nu} \partial_{\mu} \varphi \partial_{\nu} \varphi,
$$

under the assumption of spherical symmetry. ${ }^{1}$ Decomposing the $4 \mathrm{D}$ spacetime as

$$
d s^{2}=g_{\mu \nu}^{(4)} d x^{\mu} d x^{\nu}=g_{a b} d x^{a} d x^{b}+e^{-2 \phi\left(x^{a}\right)} d \Omega^{2},
$$

where $d \Omega^{2}$ is the metric on the unit two-sphere, one obtains the $2 \mathrm{D}$ action (1.1) by inserting the decomposition (1.3) into the action (1.2), imposing $\varphi=\varphi\left(x^{a}\right)$, and integrating over the angular variables. Therefore the model based on the action (1.1) seems more appropriate for discussing the quantum properties of black holes in the $s$-wave approximation than other 2D models based on the Polyakov action (describing a minimally coupled 2D massless scalar field), whose link with the real 4D world is missing. For this reason the efforts of many authors were devoted to finding the effective action which describes at the quantum level the above 2D dilaton gravity theory ([1]; see also [6] and [2]). This effective action, once derived, would allow one to go beyond the fixed background approximation usually assumed in studies of the quantum black-hole radiation discovered by Hawking [3]. Such an effective action will give in fact $\left\langle T_{a b}\right\rangle$ for an arbitrary 2D spacetime which could then be used to study selfconsistently, within this 2D approach, the backreaction of an evaporating black hole, its evolution, and its final fate. Unfortunately the effective actions so far proposed for the model of Eq. (1.1) have serious problems in correctly reproducing Hawking radiation even in a fixed Schwarzschild spacetime (see the discussion in Ref. [5]; see also [6] for a different point of view). In any case before embarking on

\footnotetext{
${ }^{1}$ The coefficient in front of the action (1.2) has been chosen in such a way that both normalization conditions and quantization rules for the 2D scalar field $\varphi$ of the action (1.1) are the standard ones in two dimensions.
} 
ambitious backreaction calculations and taking seriously puzzling results (such as antievaporation [4]) one should check for any candidate of the effective action that leads, at least for the Schwarzschild black hole, to the correct results. But what are the exact $\left\langle T_{a b}\right\rangle$ for a scalar field described by the action (1.1) propagating in a 2D Schwarzschild spacetime that the relevant effective action should predict? The aim of this paper is to partially answer this question.

By standard canonical quantization we will be able to give the asymptotic (at infinity and near the black hole horizon) values of $\left\langle T_{a b}\right\rangle$ in the three quantum states relevant for a field in the Schwarzschild spacetime, namely the Boulware state (vacuum polarization around a static star), the Unruh state (black hole evaporation), and the Hartle-Hawking state (black hole in thermal equilibrium). We will also obtain approximate analytical expressions for $\left\langle T_{a b}\right\rangle$ for every value of the radial coordinate. Any effective action for the model of Eq. (1.1) which is unable to predict at least the above asymptotic values of $\left\langle T_{a b}\right\rangle$ is incorrect (or better incomplete) and any result based on it has no physical support.

\section{II. $\left\langle T_{a b}\right\rangle$ : ASYMPTOTIC BEHAVIOR}

Our main goal is the evaluation of the renormalized expectation values of the stress tensor operator for the scalar field $\varphi$ whose dynamics is given by the action (1.1). Here we will be interested in the asymptotic values (at infinity and near the horizon). The following derivation is just a readaptation to our model of Sec. VI of the seminal paper by Christensen and Fulling [7] to which we refer the reader (see also [8]).

The classical stress tensor is defined as

$$
T_{a b}=-\frac{2}{\sqrt{-g}} \frac{\delta S}{\delta g^{a b}},
$$

and hence, from Eq. (1.1),

$$
T_{a b}=e^{-2 \phi}\left[\partial_{a} \varphi \partial_{b} \varphi-\frac{1}{2} g_{a b}(\nabla \varphi)^{2}\right] .
$$

The scalar field obeys the field equation

$$
\nabla^{a}\left(e^{-2 \phi} \nabla_{a} \varphi\right)=0 .
$$

The quantum field operator $\hat{\varphi}$ is then expanded on a basis $\left\{u_{j}\right\}$ for the solution of Eq. (2.3) in terms of annihilation and creation operators,

$$
\hat{\varphi}=\sum_{j}\left(\hat{a}_{j} u_{j}+\hat{a}_{j}^{\dagger} u_{j}^{*}\right)
$$

where $\left[\hat{a}_{i}, \hat{a}_{j}^{\dagger}\right]=\delta_{i j}$ etc. Computing the mean value $\left\langle 0\left|T_{a b}\right| 0\right\rangle$ we have

$$
\left\langle T_{a b}\right\rangle=\sum_{j} T_{a b}\left[u_{j}, u_{j}^{*}\right],
$$

where
$T_{a b}\left[u_{j}, u_{j}^{*}\right]=e^{-2 \phi}\left\{\operatorname{Re}\left[\left(\nabla_{a} u_{j}\right)\left(\nabla_{b} u_{j}^{*}\right)\right]-(1 / 2) g_{a b}\left|\nabla u_{j}\right|^{2}\right\}$.

Taking as the background geometry the exterior Schwarzschild solution

$$
d s^{2}=-(1-2 M / r) d t^{2}+(1-2 M / r)^{-1} d r^{2}, \quad \phi=-\ln r,
$$

one finds that a set of normalized basis functions of the field equation (2.3) is given by

$$
\begin{aligned}
& \vec{u}_{w}(x)=\frac{1}{\sqrt{4 \pi w}} \frac{\vec{R}(r ; w)}{r} e^{-i w t}, \\
& \overleftarrow{u}_{w}(x)=\frac{1}{\sqrt{4 \pi w}} \frac{\overleftarrow{R}(r ; w)}{r} e^{-i w t},
\end{aligned}
$$

where the radial functions $R(r ; w)$ satisfy the differential equation

$$
-\frac{d^{2} R}{d r^{* 2}}+(1-2 M / r)\left[\frac{2 M}{r^{3}}\right] R-w^{2} R=0,
$$

and $r^{*}$ is the Regge-Wheeler coordinate:

$$
r^{*}=r+2 M \ln (r / 2 M-1) .
$$

Exact solutions of Eq. (2.10) are not known; however, one can find their asymptotic behavior near the horizon,

$$
\begin{aligned}
& \vec{R} \sim e^{i w r^{*}}+\vec{A}(w) e^{-i w r^{*}}, \\
& \overleftarrow{R} \sim \overleftarrow{B}(w) e^{-i w r^{*}},
\end{aligned}
$$

and at infinity,

$$
\begin{aligned}
& \vec{R} \sim \vec{B}(w) e^{i w r^{*}}, \\
& \overleftarrow{R} \sim e^{-i w r^{*}}+\overleftarrow{A}(w) e^{i w r^{*}} .
\end{aligned}
$$

$A$ and $B$ are the reflection and transmission coefficients (see Ref. [9]).

The $\left\langle T_{a b}\right\rangle$ calculated for these modes corresponds to the so-called Boulware vacuum:

$$
\left\langle B\left|T_{a}{ }^{b}\right| B\right\rangle_{\text {unren }}=\int_{0}^{\infty} d w\left\{T_{a}{ }^{b}\left[\overleftarrow{u}_{w}, \overleftarrow{u}_{w}^{*}\right]+T_{a}{ }^{b}\left[\vec{u}_{w}, \vec{u}_{w}^{*}\right]\right\} .
$$

For the Unruh vacuum we have

$$
\begin{aligned}
\left\langle U\left|T_{a}{ }^{b}\right| U\right\rangle_{\text {unren }}= & \int_{0}^{\infty} d w\left\{T_{a}{ }_{a}\left[\overleftarrow{u}_{w}, \check{u}_{w}^{*}\right]\right. \\
& \left.+\operatorname{coth}(4 \pi M w) T_{a}^{b}\left[\vec{u}_{w}, \vec{u}_{w}^{*}\right]\right\},
\end{aligned}
$$

whereas for the Hartle-Hawking state, 


$$
\begin{aligned}
\left\langle H\left|T_{a}{ }^{b}\right| H\right\rangle_{\text {unren }}= & \int_{0}^{\infty} d w \operatorname{coth}(4 \pi M w)\left\{T_{a}{ }_{a}\left[\overleftarrow{u}_{w}, \check{u}_{w}^{*}\right]\right. \\
& \left.+T_{a}{ }^{b}\left[\vec{u}_{w}, \vec{u}_{w}^{*}\right]\right\} .
\end{aligned}
$$

As they stand these expressions are ill defined and need to be regularized. However, taking into account the regularity of the renormalized expectation values $\left\langle H\left|T_{a b}\right| H\right\rangle$ on the horizon and the vanishing of $\left\langle B\left|T_{a b}\right| B\right\rangle$ as $r \rightarrow \infty$, some asymptotic expressions can be obtained without recursion to any regularization procedure. For example for $r \rightarrow \infty$ we can write

$$
\begin{aligned}
\lim _{r \rightarrow \infty}\left\langle H\left|T_{a}{ }^{b}\right| H\right\rangle= & \lim _{r \rightarrow \infty}\left(\left\langle H\left|T_{a}{ }^{b}\right| H\right\rangle-\left\langle B\left|T_{a}{ }^{b}\right| B\right\rangle\right) \\
= & \lim _{r \rightarrow \infty}\left(\left\langle H\left|T_{a}{ }^{b}\right| H\right\rangle-\left\langle B\left|T_{a}{ }^{b}\right| B\right\rangle\right)_{\text {unren }} \\
= & \lim _{r \rightarrow \infty} 2 \int_{0}^{\infty} \frac{d w}{e^{8 \pi M w}-1}\left\{T_{a}{ }^{b}\left[\vec{u}_{w}, \vec{u}_{w}^{*}\right]\right. \\
& \left.+T_{a}{ }^{b}\left[\overleftarrow{u}_{w}, \overleftarrow{u}_{w}^{*}\right]\right\} .
\end{aligned}
$$

Similarly for the leading term at $r \rightarrow 2 M$ we have

$$
\begin{aligned}
\lim _{r \rightarrow 2 M}\left\langle B\left|T_{a}{ }^{b}\right| B\right\rangle & \sim \lim _{r \rightarrow 2 M}\left(\left\langle B\left|T_{a}{ }^{b}\right| B\right\rangle-\left\langle H\left|T_{a}{ }^{b}\right| H\right\rangle\right) \\
& =\lim _{r \rightarrow 2 M}\left(\left\langle B\left|T_{a}{ }^{b}\right| B\right\rangle-\left\langle H\left|T_{a}{ }^{b}\right| H\right\rangle\right)_{\text {unren }} .
\end{aligned}
$$

For the Unruh vacuum we have

$$
\begin{aligned}
\lim _{r \rightarrow 2 M}\left\langle U\left|T_{a}{ }^{b}\right| U\right\rangle & \sim \lim _{r \rightarrow 2 M}\left(\left\langle U\left|T_{a}{ }^{b}\right| U\right\rangle-\left\langle H\left|T_{a}{ }^{b}\right| H\right\rangle\right) \\
& =\lim _{r \rightarrow 2 M}\left(\left\langle U\left|T_{a}{ }^{b}\right| U\right\rangle-\left\langle H\left|T_{a}{ }^{b}\right| H\right\rangle\right)_{\text {unren }} \\
& =\lim _{r \rightarrow 2 M}\left\{-2 \int_{0}^{\infty} \frac{d w}{e^{8 \pi M w}-1} T_{a}{ }^{b}\left[\overleftarrow{u}_{w}, \overleftarrow{u}_{w}^{*}\right]\right\}
\end{aligned}
$$

and

$$
\begin{aligned}
\lim _{r \rightarrow \infty}\left\langle U\left|T_{a}{ }^{b}\right| U\right\rangle & =\lim _{r \rightarrow \infty}\left(\left\langle U\left|T_{a}{ }^{b}\right| U\right\rangle-\left\langle B\left|T_{a}{ }^{b}\right| B\right\rangle\right) \\
& =\lim _{r \rightarrow \infty}\left(\left\langle U\left|T_{a}{ }^{b}\right| U\right\rangle-\left\langle B\left|T_{a}{ }^{b}\right| B\right\rangle\right)_{\text {unren }} \\
& =\lim _{r \rightarrow \infty} 2 \int_{0}^{\infty} \frac{d w}{e^{8 \pi M w}-1} T_{a}{ }_{a}\left[\vec{u}_{w}, \vec{u}_{w}^{*}\right] .
\end{aligned}
$$

In deriving the above expressions we used the fact that the differences between unrenormalized and renormalized quantities are the same. This because the divergences, being ultraviolet, are state independent; hence the counterterms are the same for every state. One sees that the basic quantity entering all the expressions is $T_{a b}\left[u_{w}, u_{w}^{*}\right]$ which using the decomposition, Eqs. (2.8), (2.9), can be written in $\left(t, r^{*}\right)$ coordinates as

$$
T_{a}^{b}\left[u_{w}, u_{w}^{*}\right]=E\left(\begin{array}{cc}
-1 & 0 \\
0 & 1
\end{array}\right)+F\left(\begin{array}{cc}
0 & -1 \\
1 & 0
\end{array}\right),
$$

where

$$
\begin{aligned}
E= & \frac{1}{8 \pi w f}\left\{\left[w^{2}|R|^{2}+\frac{d R}{d r^{*}} \frac{d R^{*}}{d r^{*}}\right]-\frac{f}{r}\left(R \frac{d R^{*}}{d r^{*}}+R^{*} \frac{d R}{d r^{*}}\right)\right. \\
& \left.+|R|^{2} \frac{f^{2}}{r^{2}}\right\}
\end{aligned}
$$

and

$$
F=-\frac{i}{8 \pi f}\left(R^{*} \frac{d R}{d r^{*}}-R \frac{d R^{*}}{d r^{*}}\right)
$$

with $f \equiv(1-2 M / r)$. Using the asymptotic expansions, Eqs. (2.12),(2.13), for the radial function the limiting behaviors of $\left\langle T_{a b}\right\rangle$ can be evaluated.

Let us start by discussing what is perhaps the most interesting quantity, namely the Hawking flux for this theory, whose value has been the object of a lively debate. Only for the Unruh state is there a nonvanishing component of the flux $T_{r *}{ }^{t}$. Note also that the Wronskian contained in $F$ is constant, so it can be calculated for all $r$ from the asymptotic expansion. We find, therefore,

$$
\begin{aligned}
\left\langle U\left|T_{r^{*}}^{t}\right| U\right\rangle & =\left\langle U\left|T_{r^{*}}^{t}\right| U\right\rangle-\left\langle B\left|T_{r^{*}}^{t}\right| B\right\rangle \\
& =\left(\left\langle U\left|T_{r^{*}}^{t}\right| U\right\rangle-\left\langle B\left|T_{r^{*}}^{t}\right| B\right\rangle\right)_{\text {unren }}=f^{-1} \dot{E}_{U},
\end{aligned}
$$

where

$$
\dot{E}_{U}=\frac{1}{2 \pi} \int_{0}^{\infty} \frac{w d w}{e^{8 \pi M w}-1}|B(w)|^{2}
$$

is the energy flux at infinity. Not surprisingly, this flux is positive; i.e., there is no antievaporation of the black hole in this theory. We can calculate the total flux using Page's result [10] for the $w \rightarrow 0$ asymptotics of the greybody factor $|B(w)|^{2}$ for the $l=0$ mode:

$$
|B(w)|^{2}=16 M^{2} w^{2} .
$$

Integration over the frequencies leads to the approximate Hawking flux in this 2D theory:

$$
\dot{E}_{U}^{\text {Page }}=\frac{1}{7680 \pi M^{2}} .
$$

This low-frequency approximation for the transmission amplitude should work quite well since high frequencies will 
not contribute to the flux because of the Planckian exponent. Note that the value of the Hawking flux $\dot{E}_{U}^{\text {Page }}$ is exactly $1 / 10$ of the corresponding value coming from the Polyakov theory (massless minimally coupled 2D scalar field). This damping is due to the potential barrier present in the radial equation (2.10) which reflects the coupling of the scalar field with the dilaton. In the Polyakov theory there is no potential barrier, and hence $|B(w)|^{2} \equiv 1$ and $\dot{E}_{U}^{\text {Polyakov }}=10 \dot{E}_{U}^{\text {Page }}$.

Accurate numerical calculations of the greybody factor for $l=0$ mode and the corresponding Hawking flux give

$$
\dot{E}_{U}^{\text {numerical }}=C \dot{E}_{U}^{\text {Page }},
$$

where the coefficient

$$
C \approx 1.62 \text {. }
$$

It is interesting to compare the 2D (s-mode) Hawking flux with that of the 4D black hole. DeWitt [9] provides an approximate formula for the transmission coefficient, $|B(w)|^{2}$ $=27 M^{2} w^{2}$, which takes into account the contribution to the 4D Hawking flux of all momenta (this gives $C=1.69$ ), whereas numerical calculations [11] of the 4D Hawking flux at infinity give $\dot{E}_{U}^{4 D \text {-numerical }} \approx 1.79 \dot{E}_{U}^{\text {Page }}$.

Using the asymptotic expansion we can extract the leading behavior of $\left\langle U\left|T_{a}{ }^{b}\right| U\right\rangle$ near the horizon and at infinity [see Eqs. (2.19),(2.20)]:

$$
\left\langle U\left|T_{a}{ }^{b}\right| U\right\rangle_{r \rightarrow 2 M} \sim \frac{1}{7680 \pi M^{2}}\left(\begin{array}{cc}
1 / f & -1 \\
1 / f^{2} & -1 / f
\end{array}\right)
$$

and

$$
\left\langle U\left|T_{a}{ }^{b}\right| U\right\rangle_{r \rightarrow \infty} \sim \frac{1}{7680 \pi M^{2}}\left(\begin{array}{cc}
-1 & -1 \\
1 & 1
\end{array}\right),
$$

where now $a, b=r, t$. From Eq. (2.30) one sees the negative energy flux entering the black hole horizon which compensates the Hawking radiation at infinity.

Using similar methods one obtains [see Eqs. (2.17), (2.18)]

$$
\left\langle B\left|T_{a}^{b}\right| B\right\rangle_{r \rightarrow 2 M} \sim \frac{1}{384 \pi M^{2} f}\left(\begin{array}{cc}
1 & 0 \\
0 & -1
\end{array}\right)
$$

and

$$
\left\langle H\left|T_{a}^{b}\right| H\right\rangle_{r \rightarrow \infty} \sim \frac{1}{384 \pi M^{2}}\left(\begin{array}{cc}
-1 & 0 \\
0 & 1
\end{array}\right) .
$$

This last equation shows clearly that the Hartle-Hawking state asymptotically describes a thermal bath of $2 \mathrm{D}$ radiation at the Hawking temperature $T_{H}=(8 \pi M)^{-1}$. The prefactor is the expected $(\pi / 6) T_{H}^{2}$. This is indeed the leading contribution (in a $1 / r$ expansion) for the $s$ mode in flat space (see the Appendix).

\section{III. $\left\langle T_{a b}\right\rangle$ : ANALYTICAL APPROXIMATIONS FOR THE BOULWARE AND HARTLE-HAWKING STATES}

To obtain an analytical expression for $\left\langle T_{a b}\right\rangle$ valid for every $r \quad(2 M<r<\infty)$ we use point-splitting regularization followed by a WKB approximation for the modes. The renormalized expression $\left\langle T_{a b}\right\rangle$ is then obtained by subtraction of renormalization counterterms $\left\langle T_{a b}\right\rangle_{D S}$ coming from the DeWitt-Schwinger expansion of the Feynman Green function and removal of the regulator (point separation). This method is nicely explained in the seminal work of Anderson et al. [12] on $\left\langle T_{\mu \nu}\right\rangle$ in spherically symmetric static spacetimes, to which we refer the reader for all details. This section is just an application of their general method to our (much simpler) $s$-wave case. Here we just outline the main points of the derivation.

One first analytically continues the spacetime metric into an Euclidean form by letting $\tau=i t$ :

$$
d s^{2}=f d \tau^{2}+f^{-1} d r^{2}
$$

By the point-splitting method $\left\langle T_{a b}\right\rangle_{\text {unren }}$ is calculated by taking derivatives of the quantity $\left\langle\varphi(x) \varphi\left(x^{\prime}\right)\right\rangle$ and then letting $x^{\prime} \rightarrow x$. When the points are separated one can show that

$$
\begin{aligned}
\left\langle T_{a b}\right\rangle_{\mathrm{unren}}= & e^{-\left[\phi(x)+\phi\left(x^{\prime}\right)\right]\left[\frac{1}{2}\left(g_{a}^{c^{\prime}} G_{E ; c^{\prime} b}+g_{b}^{c^{\prime}} G_{E ; a c^{\prime}}\right)\right.} \\
& \left.-\frac{1}{2} g_{a b} g^{c d^{\prime}} G_{E ; c d^{\prime}}\right]
\end{aligned}
$$

where $G_{E}$ is the Euclidean Green function satisfying the equation

$$
\nabla^{a}\left[e^{-2 \phi} \nabla_{a} G_{E}\left(x, x^{\prime}\right)\right]=-g^{-1 / 2}(x) \delta^{2}\left(x, x^{\prime}\right),
$$

and the quantities $g_{a}^{c^{\prime}}$ are the bivectors of parallel transport. The integral representation for $G_{E}\left(x, x^{\prime}\right)$ used by Anderson et al. [12] is the following:

$$
G_{E}\left(x, x^{\prime}\right)=\int d \mu \cos \left[\omega\left(\tau-\tau^{\prime}\right)\right] p_{\omega}\left(r_{<}\right) q_{\omega}\left(r_{>}\right),
$$

where, for an arbitrary function $F$,

$$
\int d \mu F(\omega) \equiv \frac{1}{4 \pi} \int_{0}^{\infty} d \omega F(\omega)
$$

if $T=0$ (Boulware state), whereas, for $T>0$,

$$
\int d \mu F(\omega) \equiv 2 T \sum_{n=1}^{\infty} F\left(\omega_{n}\right)+T F(0)
$$

and $\omega_{n}=2 \pi n T$.

The modes $p_{\omega}$ and $q_{\omega}$ are analogous to the radial functions $\overleftarrow{R} / r, \vec{R} / r$ used in the previous section. They satisfy the Euclidean version of Eq. (2.10), which we write as 


$$
f \frac{d^{2} S}{d r^{2}}+\frac{2}{r}\left(1-\frac{M}{r}\right) \frac{d S}{d r}-\frac{\omega^{2}}{f} S=0
$$

and the Wronskian condition

$$
C_{\omega}\left[p_{\omega} \frac{d q_{\omega}}{d r}-q_{\omega} \frac{d p_{\omega}}{d r}\right]=-\frac{1}{f r^{2}}
$$

To express these modes we use the WKB approximation

$$
\begin{aligned}
& p_{\omega} \equiv \frac{1}{r \sqrt{2 W(r)}} \exp \left[\int \frac{r W(r)}{f} d r\right], \\
& q_{\omega} \equiv \frac{1}{r \sqrt{2 W(r)}} \exp \left[-\int \frac{r W(r)}{f} d r\right] .
\end{aligned}
$$

By this change of variables one sees that the Wronskian condition is satisfied by $C_{\omega}=1$. Substituting Eqs. (3.7) into the mode equation (3.5) one finds that the function $W(r)$ has to satisfy

$$
W^{2}=\omega^{2}+V+\frac{f}{2 W}\left[f \frac{d^{2} W}{d r^{2}}+\frac{d f}{d r} \frac{d W}{d r}-\frac{3 f}{2 W}\left(\frac{d W}{d r}\right)^{2}\right]
$$

where $V=(f / r) d f / d r$. This is solved iteratively starting from the zeroth-order solution

$$
W=\omega .
$$

By this method one obtains an explicit form for the modes $p_{w}, q_{w}$ to be inserted in the general expression of $G_{E}$ [Eq. (3.4)]. Taking derivatives of the latter quantity as indicated in Eq. (3.2) one eventually arrives at the following expression for $\left\langle T_{a}^{b}\right\rangle_{\text {unren }}$ :

$$
\begin{aligned}
& \left\langle T_{t}^{t}\right\rangle_{\text {unren }}=-\left\langle T_{r}^{r}\right\rangle_{\text {unren }} \\
& =e^{-2 \phi} \int d \mu \cos \left(\omega \epsilon_{\tau}\right)\left[-\frac{1}{2} g^{t t^{\prime}} \omega^{2} A_{1}-\frac{1}{2} g^{r r^{\prime}} A_{2}\right] \\
& +e^{-2 \phi} i \int d \mu \omega \sin \left(\omega \epsilon_{\tau}\right)\left[-\frac{1}{2} g^{r t^{\prime}} A_{3}\right. \\
& \left.-\frac{1}{2} g^{t r^{\prime}} A_{4}\right]
\end{aligned}
$$

where

$$
\begin{gathered}
A_{1}=p_{\omega} q_{\omega}, \quad A_{2}=\frac{d p_{\omega}}{d r} \frac{d q_{\omega}}{d r}, \quad A_{3}=q_{\omega} \frac{d p_{\omega}}{d r}, \\
A_{4}=p_{\omega} \frac{d q_{\omega}}{d r},
\end{gathered}
$$

and $\epsilon_{\tau} \equiv \tau-\tau^{\prime}$. For the sake of convenience the points are split in time only so that $r^{\prime}=r$.
The expansion for the bivectors is

$$
\begin{aligned}
& g^{t t^{\prime}}=-\frac{1}{f}-\frac{f^{\prime 2}}{8 f} \epsilon^{2}+O\left(\epsilon^{4}\right), \\
& g^{t r^{\prime}}=-g^{r^{\prime} t}=-\frac{f^{\prime}}{2} \epsilon+O\left(\epsilon^{3}\right),
\end{aligned}
$$

$$
g^{r r^{\prime}}=f+\frac{f^{\prime 2} f}{8} \epsilon^{2}+O\left(\epsilon^{4}\right)
$$

where $f^{\prime} \equiv d f / d r$.

Eventually one arrives at the following expression for $\left\langle T_{t}^{t}\right\rangle_{\text {unren }}$ in the zero temperature case:

$$
\begin{aligned}
\left\langle B\left|T_{t}{ }^{t}\right| B\right\rangle & =-\left\langle B\left|T_{r}{ }^{r}\right| B\right\rangle \\
& =\frac{1}{2 \pi f}\left[\frac{1}{\epsilon^{2}}+\frac{M^{2}}{2 r^{4}}+\frac{f^{2}}{4 r^{2}}\left[2 \gamma+\ln \left(4 \lambda^{2} \epsilon^{2}\right)\right]\right],
\end{aligned}
$$

which shows $1 / \epsilon^{2}$ and $\ln \epsilon$ divergences as $\epsilon \rightarrow 0$ ( $\lambda$ is a lower limit cutoff in the integral over $\omega$ and $\gamma$ is the Euler constant). To obtain the renormalized expressions one needs to subtract from the above expressions the renormalization counterterm $\left\langle T_{a}{ }^{b}\right\rangle_{D S}$ obtained using the following Green function (see [15] for the details):

$$
\begin{aligned}
G^{(1)}\left(x, x^{\prime}\right)= & \frac{e^{\phi(x)+\phi\left(x^{\prime}\right)}}{2 \pi}\left\{-\left[\gamma+\frac{1}{2} \ln \left(\frac{m^{2} \sigma}{2}\right)\right]\right. \\
& \left.\times\left[1+\left(\frac{R}{12}-\frac{a_{1}}{2}\right) \sigma\right]+\frac{a_{1}}{2 m^{2}}+\cdots\right\},
\end{aligned}
$$

where $m^{2}$ is an infrared cutoff and $a_{1}$ is the DeWittSchwinger coefficient for the action (1.1),

$$
a_{1}=\frac{1}{6}\left[R-6(\nabla \phi)^{2}+6 \square \phi\right]
$$

Here $R$ is the Ricci scalar for the 2D metric and $\sigma$ is one-half of the square of the distance between the points $x$ and $x^{\prime}$ along the shortest geodesic connecting them. For our splitting,

$$
\begin{gathered}
\sigma^{t}=\sigma^{; t}=\epsilon+\frac{f^{\prime 2}}{24} \epsilon^{3}+O\left(\epsilon^{5}\right), \\
\sigma^{r}=\sigma^{; r}=-\frac{f^{\prime} f}{4} \epsilon^{2}+O\left(\epsilon^{4}\right),
\end{gathered}
$$

and $\sigma=\sigma^{a} \sigma_{a} / 2$. This allows the counterterm to be evaluated in an $\epsilon$ expansion: 


$$
\begin{aligned}
\left\langle T_{t}{ }_{t}\right\rangle_{D S}= & \frac{1}{2 \pi f}\left[\frac{1}{\epsilon^{2}}+\frac{5}{12} \frac{M^{2}}{r^{4}}+\frac{1}{6} \frac{f M}{r^{3}}+\frac{f^{2}}{4 r^{2}}\right. \\
& \times\left[2 \gamma+\ln \left(m^{2} \epsilon^{2} f\right)\right], \\
\left\langle T_{r}^{r}\right\rangle_{D S}= & \frac{1}{2 \pi f}\left[-\frac{1}{\epsilon^{2}}-\frac{5}{12} \frac{M^{2}}{r^{4}}+\frac{f M}{2 r^{3}}-\frac{f^{2}}{4 r^{2}}\right. \\
& \times\left[2 \gamma+\ln \left(m^{2} \epsilon^{2} f\right)\right] .
\end{aligned}
$$

The renormalized expectation value is then defined as

$$
\left\langle T_{a b}\right\rangle=\operatorname{Re}\left[\lim _{\epsilon \rightarrow 0}\left(\left\langle T_{a b}\right\rangle_{\text {unren }}-\left\langle T_{a b}\right\rangle_{D S}\right)\right] .
$$

In the Boulware state this yields

$$
\begin{aligned}
& \left\langle B\left|T_{t}{ }^{t}\right| B\right\rangle_{W K B}=\frac{1}{2 \pi f}\left[\frac{1}{12} \frac{M^{2}}{r^{4}}-\frac{1}{6} \frac{f M}{r^{3}}-\frac{f^{2}}{4 r^{2}} \ln \left(\frac{m^{2} f}{4 \lambda^{2}}\right)\right], \\
& \left\langle B\left|T_{r}{ }^{r}\right| B\right\rangle_{W K B}=\frac{1}{2 \pi f}\left[-\frac{1}{12} \frac{M^{2}}{r^{4}}-\frac{1}{2} \frac{f M}{r^{3}}+\frac{f^{2}}{4 r^{2}} \ln \left(\frac{m^{2} f}{4 \lambda^{2}}\right)\right] .
\end{aligned}
$$

Note that $\left\langle B\left|T_{a b}\right| B\right\rangle$ has the correct trace anomaly:

$$
\begin{aligned}
\left\langle B\left|T_{a}^{a}\right| B\right\rangle_{W K B} & =\frac{a_{1}}{4 \pi}=\frac{1}{24 \pi}\left[R-6(\nabla \phi)^{2}+6 \square \phi\right] \\
& =-\frac{1}{24 \pi}\left(\frac{d^{2} f}{d r^{2}}+\frac{6}{r} \frac{d f}{d r}\right)=-\frac{M}{3 \pi r^{3}}
\end{aligned}
$$

It is easy to show that $\left\langle B\left|T_{a b}\right| B\right\rangle$ is not conserved. Reparametrization invariance of the action (1.1) gives the following nonconservation equation $[5,6]$ :

$$
\nabla_{a}\left\langle T_{b}^{a}\right\rangle=-\frac{1}{\sqrt{-g}}\left\langle\frac{\delta S}{\delta \phi} \nabla_{b} \phi\right\rangle
$$

A "source term"' is present because of the coupling with the dilaton. Equations (3.23) are nothing but the 4D conservation equations $\nabla_{\mu}\left\langle T_{\nu}^{(4) \mu}\right\rangle=0$ for the minimally coupled massless scalar field of the action (1.2). This allows us to define a " pressure" for our 2D model by rewriting Eqs. (3.23) as

$$
\begin{aligned}
8 \pi r T_{\theta}^{\theta} & =\partial_{r} T_{r}^{r}+\frac{M}{r^{2} f}\left(T_{r}^{r}-T_{t}^{t}\right), \\
\partial_{r} T_{t}^{r} & =0 .
\end{aligned}
$$

Then from Eqs. (3.20), (3.21) and (3.24) one has

$$
\left\langle B\left|T_{\theta}^{\theta}\right| B\right\rangle=\frac{1}{64 \pi^{2}}\left[\frac{8 M}{r^{5}}-\frac{2}{r^{4}}\left(1-\frac{4 M}{r}\right) \ln \left(\frac{m^{2} f}{4 \lambda^{2}}\right)\right] .
$$

It is rather interesting to note that provided we set $m=2 \lambda$ the above expressions for $\left\langle B\left|T_{a}{ }^{b}\right| B\right\rangle$ and the pressure coincide exactly with the ones derived from the "anomaly induced", effective action for the theory (1.1) [5].

The thermal case is treated similarly. Evaluating the sum over $n$ using the Plana sum formula, one finds that the stress tensor at finite temperature is obtained from the zerotemperature one by making the substitution

$$
\ln \left(\frac{m^{2} f}{4 \lambda^{2}}\right) \rightarrow\left\{2 \gamma+\ln \left(\frac{m^{2} \beta^{2} f}{16 \pi^{2}}\right)\right\}
$$

and adding the traceless pure radiation term

$$
\left(T_{t}^{t}\right)_{\mathrm{rad}}=-\left(T_{r}^{r}\right)_{\mathrm{rad}}=-\frac{\pi}{6 \beta^{2} f},
$$

where $\beta=T^{-1}$.

Summarizing, we find that in the WKB approximation for the Hartle-Hawking state,

$$
\begin{aligned}
\left\langle H\left|T_{t}{ }^{t}\right| H\right\rangle_{W K B}= & -\frac{\pi}{6 \beta^{2} f}+\frac{1}{2 \pi f}\left\{\frac{1}{12} \frac{M^{2}}{r^{4}}-\frac{1}{6} \frac{f M}{r^{3}}\right. \\
& \left.-\frac{f^{2}}{4 r^{2}}\left[2 \gamma+\ln \left(\frac{m^{2} \beta^{2} f}{16 \pi^{2}}\right)\right]\right\}, \\
\left\langle H\left|T_{r}{ }^{r}\right| H\right\rangle_{W K B}= & \frac{\pi}{6 \beta^{2} f}+\frac{1}{2 \pi f}\left\{-\frac{1}{12} \frac{M^{2}}{r^{4}}-\frac{1}{2} \frac{f M}{r^{3}}\right. \\
& \left.+\frac{f^{2}}{4 r^{2}}\left[2 \gamma+\ln \left(\frac{m^{2} \beta^{2} f}{16 \pi^{2}}\right)\right]\right\} \\
\left\langle H\left|T_{a}^{a}\right| H\right\rangle_{W K B}= & \left\langle B\left|T_{a}^{a}\right| B\right\rangle_{W K B}=-\frac{M}{3 \pi r^{3}}, \\
& \left.\times\left[2 \gamma+\ln \left(\frac{m^{2} \beta^{2} f}{16 \pi^{2}}\right)\right]\right\} \\
\langle H|P| H\rangle_{W K B}= & \frac{1}{64 \pi^{2}}\left\{\frac{8 M}{r^{5}}-\frac{2}{r^{4}}\left(1-\frac{4 M}{r}\right)\right. \\
& {\left[\frac{1}{2}\right) }
\end{aligned}
$$

where in this case $\beta=T_{H}^{-1}$.

The analytic expressions we have obtained for $\left\langle B\left|T_{a b}\right| B\right\rangle_{W K B}$ and $\left\langle H\left|T_{a b}\right| H\right\rangle_{W K B}$ have the correct asymptotic behaviors at $r \rightarrow \infty$ as inferred in the previous section. $\left\langle B\left|T_{a}{ }^{b}\right| B\right\rangle_{W K B}$ does indeed have the limiting form, Eq. (2.32), as the horizon is approached, whereas $\left\langle H\left|T_{a}{ }^{b}\right| H\right\rangle_{W K B}$ for large $r$ describes thermal radiation at the Hawking temperature in agreement with Eq. (2.33). 
In the Hartle-Hawking state the stress tensor should be regular on the horizon. This means that on the horizon the leading term of $\left\langle H\left|T_{a}{ }^{b}\right| H\right\rangle$ should be proportional to the 2D metric, since the manifold of the Euclidean instanton is regular and the Hartle-Hawking state respects all its symmetries. But the trace of the stress tensor is known exactly because we know the conformal anomaly (3.30) in $2 \mathrm{D}$. So on the horizon we should obtain

$$
\left.\left\langle H\left|T_{a}{ }^{b}\right| H\right\rangle\right|_{r=2 M}=\left.\frac{1}{2} \delta_{a}^{b}\left\langle H\left|T_{c}^{c}\right| H\right\rangle\right|_{r=2 M}=-\frac{1}{48 \pi M^{2}} \delta_{a}^{b} .
$$

In the vicinity of the horizon this provides only the leading term. Our results, Eqs. (3.28),(3.29) satisfy this condition. However, to ensure finiteness of the stress tensor near the horizon in a regular frame one should satisfy the stronger condition

$$
\frac{\left\langle H\left|T_{t}^{t}\right| H\right\rangle-\left\langle H\left|T_{r}^{r}\right| H\right\rangle}{f}=\text { finite. }
$$

This leads to serious concerns regarding the expression we found for the Hartle-Hawking state using the WKB approximation. The logarithmic term present in Eqs. (3.28),(3.29) causes $\left\langle H\left|T_{a}{ }^{b}\right| H\right\rangle_{W K B}$ to be logarithmically divergent at the horizon when calculated in a free-falling frame. This kind of logarithmic divergence is also present in the 4D calculation of Anderson et al. for non-vacuum spacetimes such as Reissner-Nordström spacetime [12]. However, numerical computations performed by the same authors give no indication that this divergence actually exists. Similarly, we suspect that the logarithmic term we have in Eqs. (3.28),(3.29) is an artifact of the WKB approximation which, as we shall see in the next section, breaks down near the horizon.

\section{IV. $\left\langle H\left|T_{a}{ }^{b}\right| H\right\rangle$ NEAR THE HORIZON}

From the discussion of the previous section one can see the disappointing fact that in the Hartle-Hawking state the energy density as measured by a free-falling observer in the WKB approximation diverges logarithmically as one approaches the horizon $r=2 \mathrm{M}$. On physical grounds we do not expect this to happen, since the Hartle-Hawking state is defined in terms of modes which are regular at the horizon. The origin of the logarithmic term in $\left\langle H\left|T_{a}{ }^{b}\right| H\right\rangle_{W K B}$ is in the counterterms $\left\langle T_{a}{ }^{b}\right\rangle_{D S}$ [see Eq. (3.18)]. The WKB approximation for the modes produces in $\left\langle T_{a}{ }^{b}\right\rangle_{\text {unren }}$, besides terms of the form $\ln \epsilon$ and and $1 / \epsilon^{2}$ which are canceled by the counterterms, only a monomial involving $f$ and powers of $r$. The natural question which arises is whether one can trust the WKB approximation near the horizon.

The Euclidean modes $Y=\left(r p_{\omega}, r q_{\omega}\right)$ [see Eq. (3.7)] satisfy a Schrödinger-like equation

$$
\begin{aligned}
\frac{d^{2} Y}{d r^{* 2}}-U\left(r^{*}\right) Y & =0, \quad U\left(r^{*}(r)\right)=\omega^{2}+V, \\
V & =\frac{2 M}{r^{3}} f, \\
f & =\left(1-\frac{2 M}{r}\right) .
\end{aligned}
$$

Solving iteratively the equation for $W^{2}$ [see Eq. (3.8)],

$$
W^{2}=\omega^{2}+V+\frac{1}{4 W^{2}} \frac{d^{2}\left(W^{2}\right)}{d r^{* 2}}-\frac{5}{16 W^{4}}\left(\frac{d\left(W^{2}\right)}{d r^{*}}\right)^{2},
$$

we get

$$
\begin{aligned}
W^{2} & =\left(W^{2}\right)_{0}+\left(W^{2}\right)_{1}+\left(W^{2}\right)_{2}+\cdots, \\
\left(W^{2}\right)_{0} & =\omega^{2}, \\
\left(W^{2}\right)_{1} & =V, \\
\left(W^{2}\right)_{2} & =\frac{1}{4\left(\omega^{2}+V\right)} \frac{d^{2} V}{d r^{* 2}}-\frac{5}{16\left(\omega^{2}+V\right)^{2}}\left(\frac{d V}{d r^{*}}\right)^{2} .
\end{aligned}
$$

Note that $V \sim f$, as do all its derivatives $\partial_{r *}^{k} V$. For $\omega=0$ the first terms $\left(W^{2}\right)_{0}$ and $\left(W^{2}\right)_{1}$ vanish at the horizon while the next "correction" $\left(W^{2}\right)_{2}$ is already finite. This indicates that the WKB approximation cannot work near the horizon for the zero-frequency mode. For the modes with non-zero $\omega$ $=\omega_{n}=(4 M)^{-1} n$ we have

$$
W^{2}=\frac{1}{(2 M)^{2}}\left[\frac{1}{4} n^{2}+f\left(1+\frac{1}{n^{2}}\right)+O\left(n^{-4}\right)\right]+O\left(f^{2}\right) .
$$

One can see that the convergence of the WKB series implies that $n$ is at least greater than 1. Evaluation of the corresponding series for $\left\langle\hat{\varphi}^{2}\right\rangle$ and the stress tensor $\left\langle H\left|T_{a}{ }^{b}\right| H\right\rangle$ near the horizon leads to exactly the same conclusion:

$$
n \gg 1 \text {. }
$$

Clearly, the standard WKB approximation cannot be applied for the calculation of the contribution of the $n=0$ and $n$ $=1$ modes to quantum averages near the horizon. To obtain a more reliable analytical expression for $\left\langle H\left|T_{a}{ }^{b}\right| H\right\rangle$ near the horizon we need a better approximation for the Green function for these modes.

In Ref. [13] it was demonstrated that a more accurate calculation of the contribution of the $n=0$ mode cures the analogous logarithmic divergence in the total $\left\langle\hat{\varphi}^{2}\right\rangle_{W K B}$. Here we follow a similar approach to analyze the stress tensor (see also [14]).

One can decompose the thermal Euclidean Green function for the $Y$ modes as 


$$
G_{E}\left(\tau, r ; \tau^{\prime}, r^{\prime}\right)=\frac{1}{\beta} \sum_{n=-\infty}^{+\infty} \frac{\cos w_{n}\left(\tau-\tau^{\prime}\right)}{\left[f(r) f\left(r^{\prime}\right)\right]^{1 / 4}} G_{n}\left(r, r^{\prime}\right),
$$

where we write $w_{n}$ for the frequency instead of just $w$ as before to make the dependence on $n$ more clear $\left(w_{n}\right.$ $=2 \pi n / \beta)$.

Near the horizon the function $G_{n}\left(r, r^{\prime}\right)$ satisfies the following differential equation (with $r \neq r^{\prime}$ ):

$$
\partial_{L}^{2} G_{n}-\left(\frac{\alpha^{2}}{M^{2}}+\frac{4 n^{2}-1}{4 L^{2}}+O(f)\right) G_{n}=0,
$$

where $L$ is defined by

$$
d L=\frac{d r}{f^{1 / 2}}
$$

and

$$
\alpha^{2}=\frac{1}{6}+\frac{n^{2}}{12}
$$

The differential equation (4.10) admits solutions in terms of Bessel functions of imaginary argument:

$$
G_{n}\left(r, r^{\prime}\right)=\left(L L^{\prime}\right)^{1 / 2} I_{n}\left(\frac{\alpha L_{<}}{M}\right) K_{n}\left(\frac{\alpha L_{>}}{M}\right) .
$$

One can show that this solution obeys the derivative condition resulting from integrating the differential equation (3.3) for $G_{E}$ across the delta function singularity at $\tau=\tau^{\prime}, r=r^{\prime}$. Using the above Green function one can calculate the corresponding contribution to the stress tensor for each $n$ near the horizon.

For a contribution to the Green function of the form

$$
e^{-i w_{n}\left(t-t^{\prime}\right)} F_{n}\left(r, r^{\prime}\right)
$$

the corresponding contribution to the unrenormalized stress tensor in the Hartle-Hawking state is

$$
\begin{aligned}
\left\langle T_{a}{ }^{b}\right\rangle_{n}= & \lim _{r \rightarrow r^{\prime}}\left\{-\frac{f}{2 r^{2}}\left[1-r\left(\partial_{r}+\partial_{r^{\prime}}\right)+r^{2} \partial_{r} \partial_{r^{\prime}}\right]\right. \\
& \left.+\frac{w_{n}^{2}}{2 f}\right\} F_{n}\left(r, r^{\prime}\right)\left(\begin{array}{cc}
1 & 0 \\
0 & -1
\end{array}\right) .
\end{aligned}
$$

For the $n=0,1,2$ modes one obtains

$$
\begin{gathered}
\left\langle T_{a}^{b}\right\rangle_{0}=\left[\frac{7 f}{240 \pi M^{2}}+O\left(f^{2}\right)\right]\left(\begin{array}{cc}
1 & 0 \\
0 & -1
\end{array}\right), \\
\left\langle T_{a}^{b}\right\rangle_{1}=\frac{1}{64 \pi M^{2}}\left[\frac{1}{f}+f(2 \gamma+\ln f)-\frac{f}{3}+O\left(f^{2}\right)\right]\left(\begin{array}{cc}
1 & 0 \\
0 & -1
\end{array}\right),
\end{gathered}
$$

$$
\left\langle T_{a}{ }^{b}\right\rangle_{2}=\left[\frac{1}{32 \pi M^{2} f}-\frac{f}{48 \pi M^{2}}+O\left(f^{2}\right)\right]\left(\begin{array}{cc}
1 & 0 \\
0 & -1
\end{array}\right) .
$$

Note that each $n>0$ contribution should be double counted to account for the $n<0$ modes as well.

These results should be compared to those coming from the WKB approximation. The $n=0$ mode does not make any contribution to $\left\langle T_{a}^{b}\right\rangle_{W K B}$ whereas the contribution of an individual mode with $n \neq 0$ is

$$
\left\langle T_{a}^{b}\right\rangle_{W K B n}=\left[\frac{|n|}{64 \pi M^{2} f}-\frac{f}{32 \pi|n| M^{2}}+O\left(f^{2}\right)\right]\left(\begin{array}{cc}
1 & 0 \\
0 & -1
\end{array}\right) .
$$

Taking the difference we find the correction to $\left\langle H\left|T_{a b}\right| H\right\rangle_{W K B}$ due to the first three modes to be

$$
\begin{aligned}
\delta\left\langle T_{a}^{b}\right\rangle_{n=0, \pm 1, \pm 2}= & {\left[\frac{f}{32 \pi M^{2}}(2 \gamma+\ln f)+\frac{17 f}{240 \pi M^{2}}\right] } \\
& \times\left(\begin{array}{cc}
1 & 0 \\
0 & -1
\end{array}\right)+O\left(f^{2}\right)
\end{aligned}
$$

Comparing this with Eqs. (3.28),(3.29) we find that the corrections above exactly cancel the logarithmic term at the event horizon to order $f \ln f$. Only the $n= \pm 1$ modes contribute such terms. For $|n|>1$ only higher-order logarithmic terms (i.e. $f^{2} \ln f$ etc.) are produced which will cause no divergence. Proceeding in a similar way we find the correction to the pressure:

$$
\begin{aligned}
\delta P_{n=0, \pm 1, \pm 2}= & \frac{1}{16 \pi M^{2}}\left[-\frac{83}{960 \pi M^{2}}-\frac{1}{32 \pi M^{2}}(2 \gamma+\ln f)\right. \\
& +O(f)]
\end{aligned}
$$

Again this cancels exactly the logarithmic term in $\langle H|P| H\rangle_{W K B}$. We can therefore conclude that for our 2D theory, Eq. (1.1), the $\left\langle H\left|T_{a}{ }^{b}\right| H\right\rangle$ and $\langle H|P| H\rangle$ are regular (in a free-falling frame) on the horizon as expected. The logarithmic term appearing in $\left\langle H\left|T_{a}{ }^{b}\right| H\right\rangle_{W K B}$ is an artifact of the WKB approximation which breaks down for the low- $n$ modes near the horizon. Furthermore, the nonlogarithmic terms in Eq. (4.20) are of order $f$, so we can obtain from Eqs. (3.28),(3.29) the following limiting values for $\left\langle H\left|T_{a}{ }^{b}\right| H\right\rangle$ on the horizon:

$$
\left\langle H\left|T_{t}{ }^{t}\right| H\right\rangle_{r=2 M}=\left\langle H\left|T_{r}{ }^{r}\right| H\right\rangle_{r=2 M}=-\frac{1}{48 \pi M^{2}} .
$$

On the other hand, the value of the pressure changes because of the first term in Eq. (4.21): 


$$
\langle H|P| H\rangle_{r=2 M}=\frac{1}{64 \pi^{2}}\left[-\frac{23}{240 M^{4}}+\frac{1}{8 M^{4}} \ln \frac{m^{2} \beta^{2}}{16 \pi^{2}}\right] .
$$

\section{CONCLUSIONS}

The main purpose of this paper was to shed some light on the rather controversial literature existing on the Hawking effect for the dilaton gravity theory described by the action (1.1). We found that the Hawking flux is manifestly positive, reduced by a greybody factor with respect to the corresponding value one gets from the Polyakov theory (no dilaton coupling). We also showed that the Hartle-Hawking state corresponds to thermal equilibrium at the Hawking temperature and that asymptotically $(r \rightarrow \infty)$ the stress tensor describes a gas of $2 \mathrm{D}$ photons. The regularity of this stress tensor on the horizon has been proved by a careful expansion of the Green function in that region eliminating the unphysical logarithmic divergence predicted by the WKB approximation. One can hope that the analogous logarithmic WKB divergence appearing in nonvacuum 4D spacetime can be handled in a similar way.

The analytic expression for $\left\langle T_{a}^{b}\right\rangle$ we found in Sec. III can be exactly reproduced by the high-frequency approximation for the effective action in static spacetimes developed by Frolov et al. [16]. This point and the generalization of our work to arbitrary curvature coupling and mass for the scalar field will be discussed elsewhere.

The feature which makes the theory (1.1) so attractive is its connection with the 4D action (1.2). What can be inferred of the physical 4D theory from the quantization of the dimensionally reduced theory we have performed? It is often said that the spherically symmetric reduced theory should describe the $s$-wave sector of the higher-dimensional one. Unfortunately in quantum field theory things are not so easy. Let us compare the value we found for the energy density in the Hartle-Hawking state on the horizon with the corresponding value coming from the quantization of the 4D theory of Eq. (1.2). Our result (which should be divided by $4 \pi r^{2}$ to restore four dimensionality) yields the following prediction for the $s$-wave contribution to the 4D theory:

$$
\left\langle H\left|T_{t}^{(s) t}\right| H\right\rangle_{r=2 M}=-\frac{1}{768 \pi^{2} M^{4}} .
$$

The value found by Anderson et al. [12] quantizing the 4D theory is

$$
\left\langle H\left|T_{t}{ }^{t}\right| H\right\rangle_{r=2 M}=\frac{1}{3840 \pi^{2} M^{4}} .
$$

The discrepancy is striking. Our 2D derived result is significantly larger than and opposite in sign to the expected 4D value. One can argue that the value of Eq. (5.2) includes the contribution of all $l$ modes and not just the $s$ one. This might be true. However, it seems unlikely that the $l>0$ modes should cancel this $l=0$ result, Eq. (5.1), to a sufficiently high degree to restore agreement with the 4D stress tensor. This difference indicates a dismal failure of the dimensional reduction. But this is not all of the story. As was shown in $[16,17]$, the $s$-mode contribution to the renormalized stressenergy tensor of the 4D theory does not coincide with the renormalized stress-energy tensor of the $2 \mathrm{D}$ reduced theory. The difference is called the dimensional-reduction anomaly. There is a suspicion that the actual mismatch between the 2D derived value, Eq. (5.1), and the 4D value, Eq. (5.2), is caused essentially by this anomaly. A preliminary analysis [18] seems to confirm this idea.

\section{ACKNOWLEDGMENTS}

This work was partly supported by the Natural Sciences and Engineering Research Council of Canada. V.F. and A.Z. are grateful to the Killam Trust for its financial support.

\section{APPENDIX A: $s$-MODE CONTRIBUTION TO THE 4D STRESS TENSOR IN FLAT SPACE AT FINITE TEMPERATURE}

In this appendix we determine the $l=0$ mode contribution to $\left\langle T_{a}{ }^{b}\right\rangle_{\beta}$ in flat space for a minimally coupled and massless 4D scalar field in a thermal state at the temperature $T$ $=\beta^{-1}$. For this case we know exactly the mode-function solutions $\varphi_{w}$ of the Klein-Gordon equation

$$
\square \varphi=0 \text {. }
$$

Insertion of the spherical decomposition

$$
\varphi=\sum_{w, l, m} \varphi_{w}(t, r) Y_{l m}(\theta, \phi)
$$

reduces Eq. (A1) to

$$
\left(-\partial_{t}^{2}+\frac{2}{r} \partial_{r}+\partial_{r}^{2}-\frac{l(l+1)}{r^{2}}\right) \varphi_{w}=0 .
$$

For the case of interest $(l=0)$ the solutions for $\varphi_{w}$ are just the ordinary Fourier modes. Taking into account that $0 \leqslant r$ $<\infty$ we must impose Dirichlet boundary conditions at $r$ $=0$. The correctly normalized $s$ modes are then

$$
\varphi_{w}=\frac{-i}{2 \pi r \sqrt{w}} e^{-i w t} \sin (w r)
$$

where $w>0$. Decomposition of the field operator $\hat{\varphi}$ in terms of the modes $\varphi_{w}$,

$$
\hat{\phi}(t, r)=\int_{0}^{\infty} d w\left[\hat{a}_{w} \varphi_{w}(t, r)+\hat{a}_{w}^{\dagger} \varphi_{w}^{*}(t, r)\right],
$$

gives the stress tensor expectation values

$$
\left\langle T_{\mu}{ }^{\nu}\right\rangle_{\beta}=\int_{0}^{\infty} d w \frac{2}{e^{\beta w}-1} T_{\mu}^{\nu}\left[\varphi_{w}, \varphi_{w}^{*}\right]
$$

where 


$$
T_{\mu \nu}\left[\varphi_{w}, \varphi_{w}^{*}\right]=\frac{1}{2}\left(\partial_{\mu} \varphi_{w} \partial_{\nu} \varphi_{w}^{*}+\partial_{\nu} \varphi_{w} \partial_{\mu} \varphi_{w}^{*}\right)-\frac{1}{2} g_{\mu \nu}\left(g^{\rho \sigma} \partial_{\rho} \varphi_{w} \partial_{\sigma} \varphi_{w}^{*}\right)
$$

Inserting Eq. (A4) into Eq. (A7) and performing the integral in Eq. (A6) we get

$$
\begin{aligned}
\left\langle T_{\mu}{ }^{\nu}\right\rangle_{\beta}= & \frac{1}{4 \pi r^{2}} \frac{\pi T^{2}}{6}\left(\begin{array}{cccc}
-1 & 0 & 0 & 0 \\
0 & 1 & 0 & 0 \\
0 & 0 & 0 & 0 \\
0 & 0 & 0 & 0
\end{array}\right)+\left(\frac{1}{32 \pi^{2} r^{4}}-\frac{T^{2}}{8 r^{2} \sinh ^{2}(2 \pi T r)}\right)\left(\begin{array}{cccc}
0 & 0 & 0 & 0 \\
0 & 0 & 0 & 0 \\
0 & 0 & -1 & 0 \\
0 & 0 & 0 & -1
\end{array}\right) \\
& +\left(\frac{T}{8 \pi r^{3}} \operatorname{coth}(2 \pi T r)-\frac{1}{16 \pi^{2} r^{4}}\right)\left(\begin{array}{cccc}
1 & 0 & 0 & 0 \\
0 & -1 & 0 & 0 \\
0 & 0 & 1 & 0 \\
0 & 0 & 0 & 1
\end{array}\right)-\frac{1}{16 \pi^{2} r^{4}} \ln \left\{\begin{array}{cccc}
1 & 0 & 0 & 0 \\
0 & -1 & 0 & 0 \\
0 & \sinh (2 \pi T r) \\
0 & 0 & 1 & 0 \\
0 & 0 & 0 & 1
\end{array}\right) .
\end{aligned}
$$

Multiplying by $4 \pi r^{2}$ and taking the limit $r \rightarrow \infty$ we obtain the result (2.33), which describes $2 \mathrm{D}$ thermal radiation at the equilibrium temperature $T=T_{H}=(8 \pi M)^{-1}$.

[1] V. Mukhanov, A. Wipf, and A. Zelnikov, Phys. Lett. B 332, 283 (1994).

[2] S. Nojiri and S. D. Odintsov, Mod. Phys. Lett. A 12, 709 (1997).

[3] S. W. Hawking, Commun. Math. Phys. 43, 199 (1975).

[4] M. Buric and V. Radovanovic, Phys. Rev. D 63, 044020 (2001).

[5] R. Balbinot and A. Fabbri, Phys. Rev. D 59, 044031 (1999); Phys. Lett. B 459, 112 (1999).

[6] W. Kummer and D. V. Vassilevich, Phys. Rev. D 60, 084021 (1999); Ann. Phys. (Leipzig) 8, 801 (1999).

[7] S. M. Christensen and S. A. Fulling, Phys. Rev. D 15, 2088 (1977).

[8] P. Candelas, Phys. Rev. D 21, 2185 (1980).

[9] B. S. DeWitt, Phys. Rep., Phys. Lett. 19C, 295 (1975).
[10] D. N. Page, Phys. Rev. D 13, 198 (1976).

[11] T. Elster, Phys. Lett. 94A, 205 (1983).

[12] P. R. Anderson, W. A. Hiscock, and D. A. Samuel, Phys. Rev. D 51, 4337 (1995).

[13] A. Tomimatsu and H. Koyama, Phys. Rev. D 61, 124010 (2000); H. Koyama, Y. Nambu, and A. Tomimatsu, Mod. Phys. Lett. A 15, 815 (2000).

[14] D. J. Rowan and G. Stephenson, J. Phys. A 9, 1261 (1976).

[15] T. S. Bunch, S. M. Christensen, and S. A. Fulling, Phys. Rev. D 18, 4435 (1978).

[16] V. Frolov, P. Sutton, and A. Zelnikov, Phys. Rev. D 61, 024021 (2000).

[17] P. Sutton, Phys. Rev. D 62, 044033 (2000).

[18] P. Sutton, Ph.D. thesis, University of Alberta, 2000. 